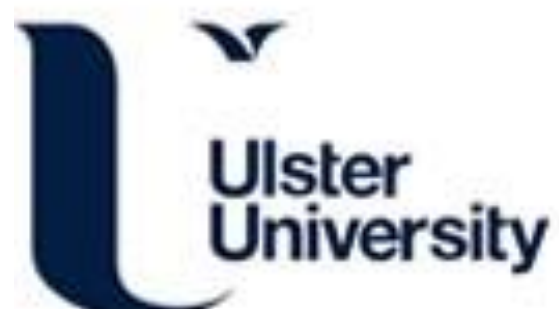

\section{Assessing retino-geniculo-cortical connectivities in Alzheimer's Disease with a neural mass model}

Bhattacharya, B., Coyle, DH., \& Maguire, L. (2011). Assessing retino-geniculo-cortical connectivities in Alzheimer's Disease with a neural mass model. In Unknown Host Publication (pp. 159-163). IEEE. https://doi.org/10.1109/CCMB.2011.5952125

Link to publication record in Ulster University Research Portal

\section{Published in:}

Unknown Host Publication

Publication Status:

Published (in print/issue): 01/01/2011

DOI:

10.1109/CCMB.2011.5952125

\section{Document Version}

Publisher's PDF, also known as Version of record

\section{General rights}

Copyright for the publications made accessible via Ulster University's Research Portal is retained by the author(s) and / or other copyright owners and it is a condition of accessing these publications that users recognise and abide by the legal requirements associated with these rights.

\section{Take down policy}

The Research Portal is Ulster University's institutional repository that provides access to Ulster's research outputs. Every effort has been made to ensure that content in the Research Portal does not infringe any person's rights, or applicable UK laws. If you discover content in the Research Portal that you believe breaches copyright or violates any law, please contact pure-support@ulster.ac.uk. 


\title{
Assessing Retino-Geniculo-Cortical Connectivities in Alzheimer's Disease with a Neural Mass Model
}

\author{
Basabdatta Sen Bhattacharya, Damien Coyle and Liam P. Maguire \\ Intelligent Systems Research Centre, University of Ulster \\ Derry BT48 7JL, Northern Ireland \\ Email: bs.bhattacharya@ulster.ac.uk; dh.coyle@ulster.ac.uk; lp.maguire@ulster.ac.uk
}

\begin{abstract}
Longitudinal studies have shown that increase of mean frequency within the theta band may be considered as an early symptom of progression into Alzheimer's Disease (AD). Also, slowing of mean frequency within the alpha band has long since been known to be a def nitive marker in AD. This work is aimed at developing a better understanding of alterations in neuronal connectivity underlying Electroencephalogram (EEG) changes in AD. Specif cally, connectivity changes in the dorsolateral geniculo-cortical pathway are studied using a neural mass computational model. Connectivity parameters in the model are informed by the most recent experimental data on mammalian Lateral Geniculate Nucleus (dorsal). A slowing of the mean power spectra of the model output is observed with increase in both excitatory and inhibitory parameters in the intra-thalamic and thalamocortical pathways and a decrease of sensory pathway synaptic connectivity. The biological plausibility of the results suggest potential of further model extension in AD research.
\end{abstract}

\section{INTRODUCTION}

Currently specif ed diagnostic symptoms for Alzheimer's Disease (AD) correspond to a stage where irreversible damage has already occurred in specif c neural pathways [1]-[3]. The biggest challenge in treatment of Alzheimer's Disease (AD) today is in fnding a biomarker that can detect the disease well in advance and prior to such irreparable pathological alterations [4]-[7]. Recent advances in this direction have suggested retinal alterations as an early indicator of $\mathrm{AD}$ [2], [8]. This is in line with impaired visuo-cognitive skills reported in Alzheimer's Disease [9], [10]. An increase in theta rhythms and decrease in alpha rhythms (commonly referred to as 'slowing of mean EEG') are well known symptoms in EEG of patients with dementia of the Alzheimer type [11], [12]. It is now believed that similar thalamocortical mechanisms underpin the generation and behaviour of both alpha and theta rhythms [13], [14]. Furthermore, slowing of the mean EEG has long since been considered as an indication of deteriorating cognitive abilities [15]-[17]. In previous research [18], [19] we have used a classic neural mass computational model (Alpha Rhythms model (ARm)) [20], [21] to study the underlying neuronal synaptic aberrations as a cause of changed EEG in AD. Our results showed a slowing of the alpha rhythm with an increase of inhibitory connectivity parameter in the model, while the excitatory connectivity parameter was held at its initial values.

More recently, we presented a modif ed version of the ARm frstly by introducing a synaptic connectivity in the retinogeniculate pathway, and secondly by introducing synaptic connectivity parameters based on experimental data from the dorsal Lateral Geniculate Nucleus (LGNd) [22]. This model was used for a similar study (as in [18], [19]) on both alpha and theta frequency bands. The model output displayed a def nite slowing of the dominant frequency within the alpha and theta bands with increasing values of the connectivity parameters in the intra-thalamic loop and decreasing values of connectivity parameter in the retino-geniculate pathway. These results, and research reports of a probable link between the retinal pathway aberrations and $\mathrm{AD}$ [2], [8]-[10], inspired us to carry out the work presented in this paper. We propose to study the the modif ed version of the model (in [22]) with an emphasis on the retino-geniculate synaptic connectivity and its impact on the model behaviour in context to EEG behaviour in AD. Furthermore, the model output is studied by a simultaneous variation of connectivity parameters, unlike a single parameter variation approach in all our earlier works. The output of the model mimics cortical EEG [20] and corresponds to EEG changes associated with synaptic alterations in the retinogeniculo-cortical pathway. We speculate that such an approach might help to identify an EEG biomarker corresponding to visual modality related def ciencies observed in AD.

In Section II, we provide an overview of the model used in this work. The results are presented in Section III. Discussion of the results and future work is mentioned in Section IV.

\section{A SIMPLE NEURAL MASS MODEL OF THE RETINO-GENICULO-CORTICAL PATHWAY}

The model used in this work is shown in Figure 1 and is based on the classic Alpha Rhythm model (ARm) originally proposed by Lopes da Silva [20] and later modif ed [21]. It has two representative neuronal populations: Thalamocortical Relay (TCR) cells of the Lateral Geniculate Nucleus (LGN) and cells of the Thalamic Reticular Nucleus (TRN). The TCR cells receive excitatory afferents from the retinal cells and inhibitory feedback from the TRN cells. The TRN cells receive excitatory feed-forward afferents from the TCR cells. The resting state retinal afferents correspond to closed eyes of a subject and is simulated by a stochastic signal $\Psi_{\mu, \varphi}(t)$ with mean $\mu$ and standard deviation $\varphi$ and expressed in spikes/sec [20], [21], [25], [26]. Excitatory and inhibitory synapses are represented 


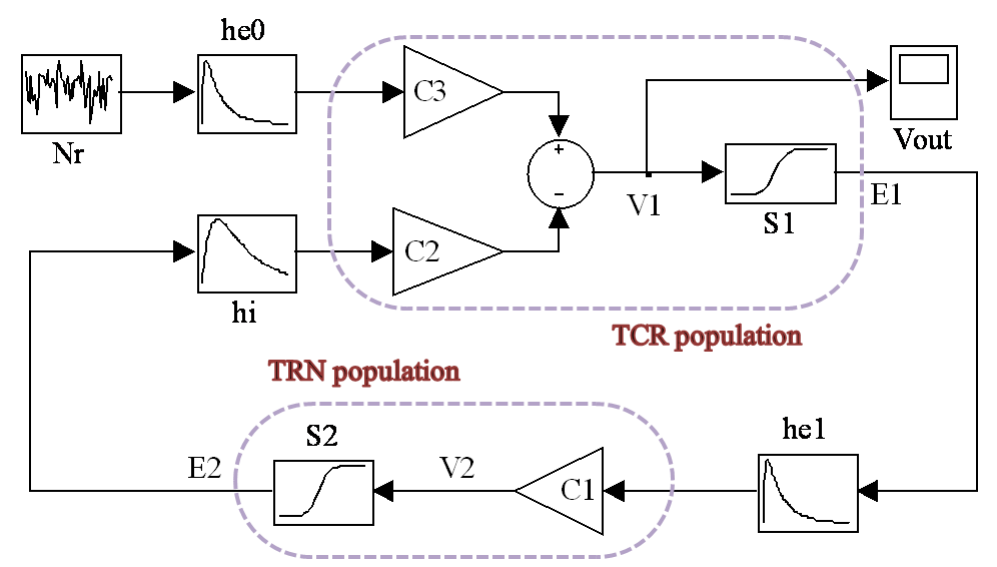

Fig. 1. The neural mass thalamocortical circuitry as a modif cation of the ARm and as simulated in our work with Simulink ${ }^{\circledR}$ in Matlab. The connectivity parameter values in the model are based on most recent experimental data (from the cat Lateral Geniculate Nucleus (LGN)) available from literature [23], [24] and represent the proportion of excitatory or inhibitory synapses from respective afferents on a single dendritic terminal of a cell. The block $N_{r}$ represents retinal input to the model in an eyes-closed resting condition, simulated by a stochastic signal $\Psi_{\mu, \varphi}(t)$ with mean $\mu$ and standard deviation $\varphi$. All other blocks in the model are def ned in (1)-(3). The parameter values for all blocks in the fgure are provided in Table I.

by a response function def ned as [25]-[28]:

$$
h_{e, i}(t)=\frac{A_{e, i}}{\tau_{e, i}} t e^{-\frac{1}{\tau_{e, i}}},
$$

where $\{e, i\}$ represent excitatory or inhibitory synapses respectively, $A$ is the synaptic amplitude and $\tau$ is the rise-time (time to reach $90 \%$ of the maximum value) of the kernel function $h_{e, i}(t)$. The 'collective' (commonly referred to as 'lumped') average membrane potential $V_{m p}(t)$ of a neuronal population is expressed as:

$$
V_{m p}(t)=h_{e, i}(t) \otimes \xi(t),
$$

where $h_{e, i}(t)$ is as def ned in (1) and $\xi(t)$ is the pre-synaptic spike rate and expressed in spikes/sec. The membrane potential at the collective soma of a neuronal population is converted by a sigmoid operator $S($.$) to an average spike rate E(t)$, representing the collective spiking frequency of the population and def ned as [26], [28], [29]:

$$
E(t)=S\left[V_{m p}(t)\right]=\frac{2 e_{0}}{1+e^{\nu\left(s_{0}-V_{m p}(t)\right)}},
$$

where $e_{0}$ is the maximum fring rate of the population, $s_{0}$ is the resting membrane potential and $\nu$ is the sigmoid steepness parameter.

We consider synaptic connectivity, unlike f bre connectivity considered in the original model [20], [21]. Furthermore, our choice of parameters is based on most recent available data obtained from cat LGN (dorsal) as reported in [23], [24]. The synaptic connectivity parameters in the model represent the proportion of a certain type of synapse on a neuronal population with respect to the total number of synapses converging on a single dendrite of the population from different sources. In Figure 1, $C_{1}$ represents the proportion of (excitatory) synapses made by the collaterals of the thalamocortical efferents from the TCR on the TRN population and is expressed as a percentage of the total number of synaptic vesicles at the synaptic junctions of a single dendrite of the population.
TABLE I

PARAMETERS USED IN THE MOdel AND DEFINED IN (1) AND (3). THE VALUES OF THE CONNECTIVITY PARAMETERS $C_{1}, C_{2}$ AND $C_{3}$ ARE AS OBTAINED FROM MOST RECENT EXPERIMENTAL DATA FROM THE CAT LGN AND REPORTED IN [23], [24]. THE VALUES OF PARAMETERS IN (1) ARE AS USED IN [26], [28] WHILE THOSE USED IN (3) ARE AS IN [25],

[27].

\begin{tabular}{lcr}
\hline Parameters & Units & Values \\
\hline \hline$\mu$ & spikes per sec (sps) & 550 \\
$\varphi$ & sps $^{2}$ & 120 \\
$A_{e}$ & $\mathrm{mV}$ & 3.25 \\
$A_{i}$ & $\mathrm{mV}$ & 22 \\
$\tau_{e}$ & $\mathrm{msec}$ & 10 \\
$\tau_{i}$ & $\mathrm{msec}$ & 20 \\
$\nu$ & $\mathrm{mV}-1$ & 0.56 \\
$e_{0}$ & $\mathrm{~V}$ & 25 \\
$s_{0}$ & $\mathrm{mV}$ & 6 \\
$C_{1}$ & \% of total (TRN) & 35 \\
$C_{2}$ & \% of total (TCR) & 28 \\
$C_{3}$ & \% of total (TCR) & 10 \\
\hline
\end{tabular}

Similarly, $C_{2}$ represents the inhibitory synapses by the TRN cells on the TCR population while $C_{3}$ represents the excitatory synapses by the retinal afferents on the TCR population. The experimental data corresponding to these synaptic connectivity parameters in the model are $-C_{1}: 30-40 ; C_{2}: 24-31$; $C_{3}: 7-12$. We refer to these values as the 'normal range' of connectivity values indicating that these are obtained from a pathologically intact $\mathrm{LGN}(\mathrm{d})$.

The output of the model is the simulated membrane potential of the TCR population and mimics occipital EEG. All parameter values are def ned in Table I. The connectivity parameter values in Table I are referred to in this work as their 'basal' values (synaptic connectivity values in a simulation of pathologically unaltered brain condition) and are evaluated as mean of the normal range of values obtained from experimental data and as mentioned above. Results are discussed in the Section III. 


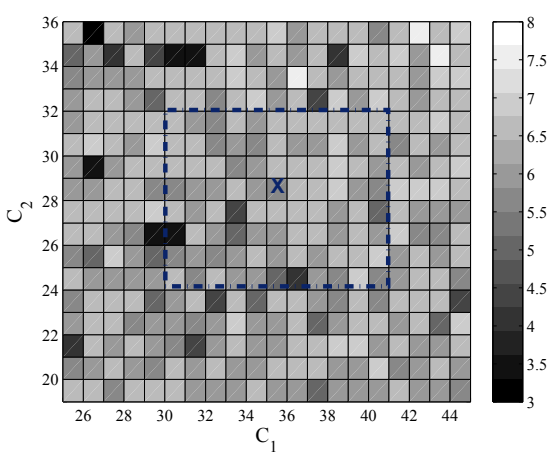

(a)

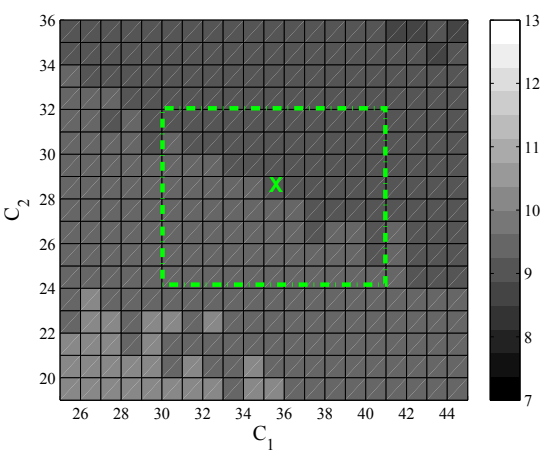

(d)

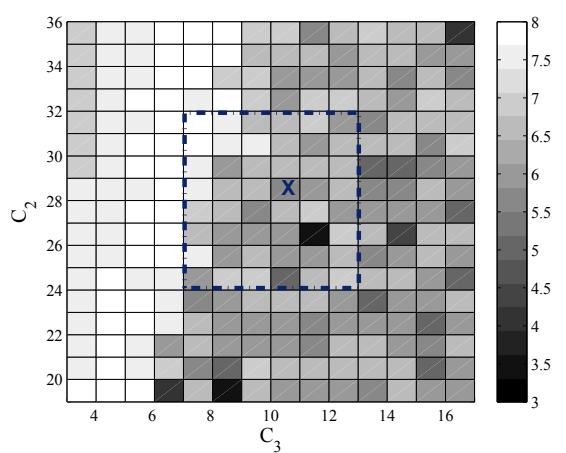

(b)

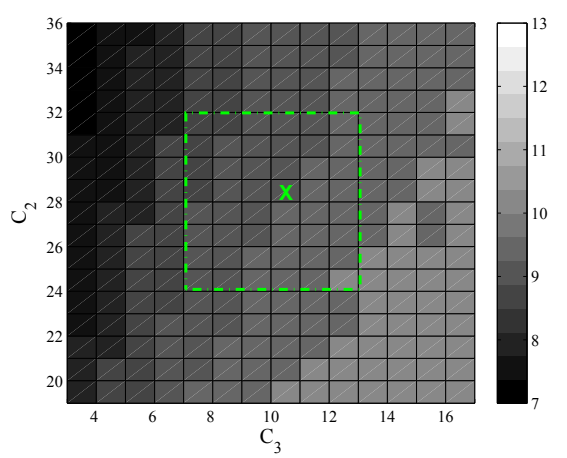

(e)

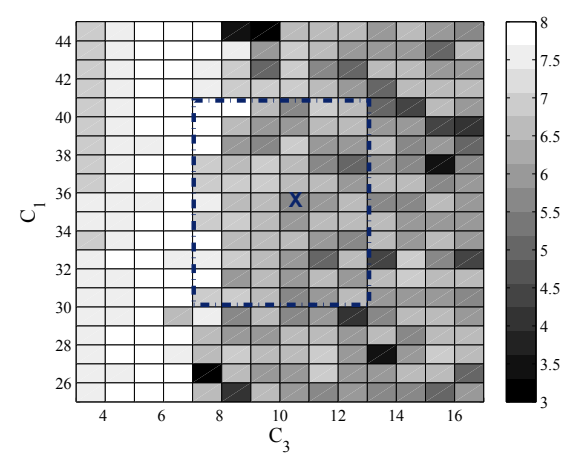

(c)

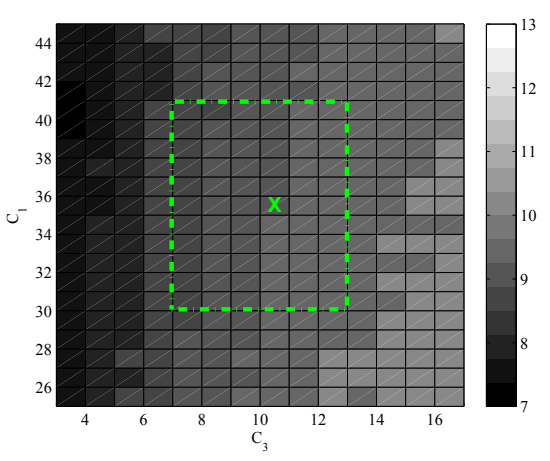

(f)

Fig. 2. Plots showing average dominant frequency behaviour of the model output within the (a) - (c) theta $(4-7 H z)$ and (d) $-(\mathrm{f})$ alpha $(7.5-13 H z)$ bands. The parameter variation ranges are $-C_{1}: 25-45 ; C_{2}: 19-36 ; C_{3}: 1-17$, while the normal range (range of values in a pathologically intact brain) for each of the parameters are $-C_{1}: 30-40 ; \quad C_{2}: 24-31 ; C_{3}: 7-12$. The basal value (a base value used to benchmark the model output oscillatory behaviour representing that of a pathologically unaltered brain) of each parameter is taken as the mean of the normal range of values for the respective parameter and is shown in Table I. The ' $\mathrm{X}$ ' in each plot identif es the output response when all three parameters are at their basal values. The region within the square in each plot show the boundaries of the normal range of values of each parameter. (a)(c) Variation of the excitatory $C_{1}$ and inhibitory $C_{2}$ parameters in the thalamocortical pathway when the excitatory parameter $C_{3}$ in the retinal afferent pathway is at its basal value 10 . (b)(e) Variation of $C_{3}$ and $C_{2}$ when $C_{1}$ is at its basal value 35. (c)(f) Variation of $C_{3}$ and $C_{1}$ when $C_{2}$ is at its basal value 24.

\section{EXPERIMENTAL METHODS AND RESULTS}

To simulate abnormal pathological conditions, the connectivity parameters in the model are varied above and below their basal values. The output power spectra of the simulated 'EEG' from the model is observed within theta $(4-7 \mathrm{~Hz})$ and alpha $(7.5-13 \mathrm{~Hz})$ frequency bands. Similar observations were made in our previous work by varying the connectivity parameters at arbitrary intervals about their basal values [18], [19]. In this work, we perform a global parameter search in order to study the dynamics of the output spectra at a higher resolution of parametric variations. Thus, we vary the connectivity parameters to every consecutive integer value within their respective normal ranges. Further, the values are varied up to \pm 5 of their maximum and minimum values respectively. The parameters of the synaptic function as well as the sigmoid function are also different than those in our previous work and are based on physiologically plausible values as reported and used in [26], [28].

Recently, we have demonstrated that the choice of input mean $\mu$ and the standard deviation $\varphi$ in this model as well as in ARm are crucial to the output oscillation frequency [19],
[22]. In this work, we adjusted these values by trial simulations so as to obtain an output dominant frequency of $10.5-11 \mathrm{~Hz}$ within the alpha band and $4-6 \mathrm{~Hz}$ within the theta band while the connectivity parameters are set at their basal values.

Model simulation is implemented using the $4^{\text {th }} / 5^{\text {th }}$ order Runge-Kutta ODE solver within the Simulink ${ }^{\circledR}$ environment in Matlab. Total simulation time is 10 seconds with a sampling frequency of $250 \mathrm{~Hz}$. The output from each simulation is clipped on the time axis so as to abstract the values from the start of the $4^{\text {th }} \mathrm{sec}$. to the end of the $8^{\text {th }} \mathrm{sec}$. as is done in experimental studies of EEG [21], [30]. The output vector thus obtained is bandpass fltered between $3-8 \mathrm{~Hz}$ (to extract the theta rhythm components) and $6.5-14 \mathrm{~Hz}$ (to extract the alpha rhythm components) using a Butterworth flter of order 10 in Matlab. The power spectra of this fltered output is computed in Matlab using a Welch periodogram with hamming window of segment length $1 / 4^{\text {th }}$ the size of the sampling frequency and overlap of $50 \%$ [18], [19], [31].

The results of variations of the connectivity parameters on the dominant frequency of the output spectra are shown in Figure 2. Each plot shows variation of model output dominant 
frequency corresponding to variation of any two parameters; we show the cases when the third parameter is at its respective basal value. Compared to the other plots, there is a clear lack of systematic change in Figure 2(a) indicating that the connectivity parameters $C_{1}$ and $C_{2}$ have very little inf uence on the output behaviour within the theta band. In contrast, the parameter $C_{3}$ seem to play a major role on the theta band output behaviour. In both Figures 2(b) and 2(c), we see that there is a sudden increase in dominant frequency within the theta band for parameter values below the normal range minimum $\left(C_{3}<7\right)$. On the other hand, the parameters $C_{1}$ and $C_{2}$ show a marked inf uence on the alpha band behaviour with a slowing within the alpha band for increasing values of $C_{2}$ observed in Figure 2(d). Furthermore, the average dominant frequency of oscillation within the alpha band is also reduced with increasing values of $C_{1}$. These results agree with our earlier work with the ARm [18], [19], in spite of change in model parameters and structure. Figures 2(e) and 2(f) show a consistent slowing of the alpha rhythm with decreasing values of $C_{3}$. The diagonal patterns in Figure 2(e) indicates an overall diminishing of the dominant frequency within the alpha band with increasing values of $C_{2}$. However, the diagonal patterns are much steeper in Figure 2(f) showing a lesser impact of $C_{1}$ on the slowing of dominant frequency, thus agreeing with Figure 2(d). Overall, Figures 2(d)-2(f) show that all the three parameters interact with one another within the alpha band.

A series of multi-way ANOVAs (done using the Statistical Toolbox ${ }^{T M}$ in Matlab) show that the effects of variation of all three parameters on the average dominant frequency, shown in Figures 2(b)- 2(f), are statistically signif cant $(P \ll 0.001)$. However, effects of variation of $C_{1}$ and $C_{2}$ on average dominant frequency within the theta band, shown in Figure 2(a), are not signif cant statistically $(P>0.001)$. The results are discussed in the following section.

\section{DisCUSSION AND FUTURE WORK}

In this work, we present a modif ed version of the Alpha Rhythm model originally proposed by Lopes da Silva, subsequently extended [21] and validated in our previous work [18], [22]. We endeavoured to use the model in studying the underlying neuronal mechanism corresponding to changes in mean EEG observed in Alzheimer's Disease patients. Here, we present a preliminary exhaustive global connectivity parameter search in order to study the model output behaviour within the theta and the alpha bands at a higher resolution than reported in our previous works.

Results presented in Section III show that there is an overall slowing of the mean dominant frequency in the output spectra when values of synaptic connectivity in the retinal pathway are below its normal range. This is indicated by the step increase in the dominant frequency within the theta band and a decrease within the alpha band. The output behaviour is thus in agreement with various research reports stating the vital role of several aspects of the visual modality as a biomarker in detection of Alzheimer's Disease [8], [10], especially in its early stages [2], [9]. Furthermore, increase in theta band power is reportedly a symptom of early stage Alzheimer's Disease [11], [32]. Since the retina is the most accessible part of the nervous system [2], [33], we speculate that such simulated results indicating underlying loss in neuronal synaptic connectivity might suggest new directions of experimental investigation in Alzheimer's Disease and possibly lead to new diagnostic and therapeutic tools.

The overall slowing of mean output frequency within the alpha band with increasing values of both the excitatory $\left(C_{1}\right)$ and inhibitory $\left(C_{2}\right)$ connectivity parameters in the thalamocortical loop is contrasting to the increase in alpha band average dominant frequency seen with increasing values of the excitatory parameter $\left(C_{3}\right)$ in the retinal pathway. This agrees with results presented in our earlier work [22] where we have hypothesised that such behaviour indicate a vital role of the inhibitory cell population in the model. As the inhibitory cells represent characteristics of the TRN cell population, the results conform to experimental reports stating the important role of the TRN in modulating thalamic oscillatory behaviour [34][36].

Although parameters used in the model presented here are based on experimental data, there is ample scope for incorporating more biological plausibility - for example, the cortico-thalamic connectivities are now known to play a vital role in the thalamic as well as cortical oscillations [37]-[40]. Furthermore, experimental data suggests that the majority of synaptic inputs to the thalamic cells are from the cortex [23], [24], [41]. Alzheimer's Disease is characterised by not only cortical, but also thalamic atrophy [42], [43]. In a recent study (under review for publication), we have shown that retinal connectivity with the thalamic inhibitory interneuron (IN) population in the model plays a more important role in slowing of alpha band power as compared to connectivity with the Thalamic Relay Cells (TCR). This is a signif cant result as f rstly, an essential role of inhibitory brain circuitry in modulating brain rhythms is re-iterated; and secondly, this gives a direction to future research where the role played by IN cell population may be investigated more thoroughly than until now. We anticipate that such a research direction may provide vital clues on the retinal and thalamic role in modulation and alteration of cortical EEG in neuro-degenerative diseases such as $\mathrm{AD}$.

\section{ACKNOWLEDGEMENT}

This work is supported by the Northern Ireland Department for Education and Learning under the 'Strengthening the All Island Research Base' programme. B. Sen Bhattacharya would like to thank Dr. David Watson for valuable comments and suggestions on the work and several useful discussions from time to time.

\section{REFERENCES}

[1] M. Eric Siemers, "Advances in biomarkers and modelling for the development of improved therapeutics: early Alzheimer's treatment," Abstract of talk at: $1^{\text {st }}$ International Congress on Alzheimer's Disease and Advanced Neurotechnologies, February 2010. 
[2] S. Frost, R. N. Martins, and Y. Kanagasingam, "Ocular biomarkers for early detection of Alzheimer's Disease," Journal of Alzheimer's Disease, vol. 22, no. 1, pp. 1-16, 2010.

[3] P. J. Nestor, P. Scheltens, and J. R. Hodges, "Advances in early detection of Alzheimer's Disease," Nature Reviews, pp. S34-S41, 2004, doi: $10.1038 / \mathrm{nm} 1433$.

[4] J. C. de la Torre, "Alzheimer's Disease is incurable but preventable," Journal of Alzheimer's disease, vol. 20, no. 3, pp. 861-870, 2010.

[5] W. H. Waugh, "A call to reduce the incidence of Alzheimer's Disease," The journal of applied research, vol. 10, no. 2, pp. 53-57, 2010.

[6] J. Dauwels, F. Vialatte, and A. Cichocki, "Diagnosis of Alzheimer's Disease from EEG signals: Where are we standing?" Current Alzheimer's Research, vol. xx, pp. 1-43, 2010.

[7] P. S. Aisen, "Alzheimer's Disease therapeutic research: the path forward," Biomed central, vol. 1, pp. 1-6, 2009.

[8] M. F. Cordeiro, K. M. Coxon, J. Duggan, S. Nizari, E. M. Normando, S. L. Sensi, A. M. Sillito, F. W. Fitzke, T. E. Salt, and S. E. Moss, "Imaging multiple phases of neurodegeneration: a novel approach to assessing cell death in vivo," Cell Death and Disease, vol. 1, pp. 1-11, 2010.

[9] W. J. Tippett and L. E. Sergio, "Visuomotor integration is impaired in early stage Alzheimer's Disease," Brain Research, vol. 1102, pp. 92 102, 2006.

[10] P. J. Uhlhaas, J. Pantel, H. Lanfermann, D. Prvulovic, C. Haenschel, K. Maurer, and D. E. J. Linden, "Visual perceptual organization def cits in Alzheimer's dementia," Dementia and Geriatric Cognitive Disorders, vol. 25, no. 5, pp. 465-475, 2008.

[11] J. Jeong, "EEG dynamics in patients with Alzheimer's Disease," Clinical Neurophysiology, vol. 115, pp. 1490-1505, 2004.

[12] H. Adeli, S. Ghosh-Dastidar, and N. Dadmehr, "Alzheimer's Disease: models of computation and analysis of EEGs," Clinical EEG and Neuroscience, vol. 36, no. 3, pp. 131-136, 2005.

[13] S. W. Hughes, M. Lorincz, D. W. Cope, K. L. Blethyn, K. A. Kekesi, H. R. Parri, G. Juhasz, and V. Crunelli, "Synchronised oscillations at $\alpha$ and $\theta$ frequencies in the Lateral Geniculate Nucleus," Neuron, vol. 42, pp. 253-268, 2004.

[14] S. W. Hughes and V. Crunelli, "Thalamocortical mechanisms in EEG alpha rhythms and their pathological implications," The Neuroscientist, vol. 11, no. 4, pp. 357-372, 2005.

[15] A. Stoller, "Slowing of the alpha-rhythm of the electroencephalogram and its association with mental deterioration and epilepsy," Journal of Mental Science, vol. 95, pp. 972-984, 1949, doi: 10.1192/bjp.95.401.972.

[16] W. Klimesch, "EEG alpha and theta oscillations ref ect cognitive and memory performance: a review and analysis," Brain Research Reviews, vol. 29, pp. 169-195, 1999.

[17] T. Montez, S. S. Poil, B. F. Jones, I. Manshanden, J. P. Verbunt, B. W. van Dijk, A. B. Brussaard, A. van Ooyen, C. J. Stam, P. Scheltens, and K. Linkenkaer-Hansen, "Altered temporal correlations in parietal alpha and prefrontal theta oscillations in early-stage Alzheimer's Disease," Proceedings of the National Academy of Science, vol. 106, no. 5, pp. 1614-1619, 2009.

[18] B. S. Bhattacharya, D. Coyle, and L. P. Maguire, "A computational modelling approach to investigate alpha rhythm slowing associated with Alzheimer's Disease," in Proceedings of the conference on Brain Inspired Cognitive Systems (BICS), Madrid, Spain, July 2010, pp. 382 392.

[19] _ - "Thalamocortical circuitry and alpha rhythm slowing: an empirical study based on a classic computational model," in Proceedings of the International Joint Conference on Neural Networks (IJCNN), Barcelona, Spain, July 2010, pp. 3912- 3918.

[20] F. H. L. da Silva, T. H. M. T. van Lierop, C. F. Schrijer, and W. S. van Leeuwen, "Essential differences between alpha rhythms and barbiturate spindles: spectra and thalamo-cortical coherences," Electroencephalography and Clinical Neurophysiology, vol. 35, pp. 641-645, 1973.

[21] P. Suffczyński, "Neural dynamics underlying brain thalamic oscillations investigated with computational models," Ph.D. dissertation, Institute of experimental physics, University of Warsaw, October 2000.
[22] B. S. Bhattacharya, D. Coyle, and L. P. Maguire, Brain Inspired Cognitive Systems 2010. Springer, 2010, ch. Alpha and theta rhythm abnormality in Alzheimer's Disease: a study using a computational model (invited book chapter, to appear), p. xx.

[23] E. G. Jones, The Thalamus, Vol. I and II, 1st ed. Cambridge, UK: Cambridge University Press, 2007.

[24] S. M. Sherman and R. W. Guillery, Exploring the thalamus, 1st ed. New York: Academic Press, 2001.

[25] B. H. Jansen, G. Zouridakis, and M. E. Brandt, "A neurophysiologicallybased mathematical model of $\mathrm{f}$ ash visual evoked potentials," Biological Cybernetics, vol. 68, pp. 275-283, 1993.

[26] O. David and K. J. Friston, "A neural mass model for MEG/EEG: coupling and neuronal dynamics," NeuroImage, vol. 20, pp. 1743-1755, 2003.

[27] B. H. Jansen and V. G. Rit, "Electroencephalogram and visual evoked potential generation in a mathematical model of coupled cortical columns," Biological Cybernetics, vol. 73, pp. 357-366, 1995.

[28] A. Babajani and H. Soltanian-Zadeh, "Integrated MEG/EEG and fMRI model based on neural masses," IEEE Transactions on Biomedical Engineering, vol. 53, no. 7, pp. 1794-1801, 2006.

[29] M. Zavaglia, L. Astolf, F. Babiloni, and M. Ursino, "A neural mass model for the simulation of cortical activity estimated from high resolution EEG during cognitive or motor tasks," Journal of Neuroscience Methods, vol. 157, pp. 317-329, 2006.

[30] C.J.Stam, J. Pijn, P.Suffczyński, and F. da Silva, "Dynamics of the human alpha rhythm:evidence for non-linearity?" Clinical Neurophysiology, vol. 110, pp. 1801-1813, 1999.

[31] J. L. Cantero, M. Atienza, G. Gomez-Herrero, A. Cruz-Vadell, E. GilNeciga, R. Rodriguez-Romero, and D. Garcia-Solis, "Functional integrity of thalamocortical circuits differentiates normal ageing from mild cognitive impairment," Human Brain Mapping, vol. 30, pp. 3944-3957, 2009.

[32] H. Adeli, S. Ghosh-Dastidar, and N. Dadmehr, "Alzheimer's Disease and models of computation: Imaging, classif cation and neural models," Journal of Alzheimer's Disease, vol. 7, no. 3, pp. 187-199, 2005.

[33] B. S. Bhattacharya, "Information recovery from rank-order encoded images," Ph.D. dissertation, School of Computer Science, University of Manchester, 2008.

[34] B. Amsallem and B. Pollin, "Possible role of the nucleus reticularis thalami (nRT) in the control of specif c, non-specif $\mathrm{c}$ thalamic nuclei and cortex activity," Pain, vol. 18, pp. S-283, 1984.

[35] F. Crick, "Function of the thalamic reticular complex: the searchlight hypothesis," Proceedings of the National Academy of Sciences, vol. 81 pp. 4586-4590, 1984.

[36] U. Kim, M. V. Sanchez-Vives, and D. A. McCormick, "Functional dynamics of gabaergic inhibition in the thalamus," Science, vol. 278, pp. 130-134, 1997.

[37] J. Wolfart, D. Debay, G. L. Masson, A. Destexhe, and T. Bal, "Synaptic background activity controls spike transfer from thalamus to cortex," Nature Neuroscience, vol. 8, no. 12, pp. 1760-1767, 2005.

[38] A. Destexhe, Computational Neuroscience in Epilepsy. Elsevier, Amsterdam, 2008, ch. Cortico-thalamic feedback: a key to explain absence seizures, pp. 184-214.

[39] S. M. Sherman and R. W. Guillery, "Thalamic relay function and their role in cortico-cortical communication: generalizations from the visual system," Neuron, vol. 33, pp. 163-175, 2002.

[40] S. M. Sherman, "Thalamic relays and cortical functioning," Progress in Brain Research, vol. 149, pp. 107-126, 2005.

[41] S. C. V. Horn, A. Erisir, and S. M. Sherman, "Relative distribution of synapses in the A-laminae of the Lateral Geniculate Nucleus of the cat," The Journal of Comparative Neurology, vol. 416, pp. 509-520, 2000.

[42] L. W. de Jong, K. van der Hiele, I. M. Veer, J. J. Houwing, R. G. J. Westendorp, E. L. E. M. Bollen, P. W. de Bruin, H. A. M. Middelkoop, M. A. van Buchem, and J. van der Grond, "Strongly reduced volumes of putamen and thalamus in alzheimer's disease: an mri study," Brain, vol. 131, pp. 3277-3285, 2008.

[43] J. H. Xuereb, R. H. Perry, J. M. Candy, E. K. Perry, E. Marshall, and J. R. Bonham, "Nerve cell loss in the thalamus in Alzheimer's Disease and Parkinson's Disease," Brain, vol. 114, pp. 1363-1379, 1991. 